\title{
Respuesta al comentario de Barbosa-Espitia et al. a "Emplazamiento del magmatismo Paleoceno-Eoceno bajo un régimen transtensional y su evolución a un equilibrio dinámico en el borde occidental de Colombia"
}

\author{
Jeny Alejandra Grajales 1,2,*, Ángel F. Nieto-Samaniego ${ }^{3}$, \\ Darío Barrero Lozano ${ }^{4}$, Jairo Alonso Osorio ${ }^{5}$ y \\ Mario Andrés Cuellar ${ }^{5}$ \\ ${ }^{1}$ Universidad de Concepción, Facultad de ciencias Químicas, \\ Edmundo Larenas 234, Campus Concepción, Chile. \\ ${ }^{2}$ La Luna E\&P S DE RL Sucursal Colombia, Carrera 13A No. 87-17, Bogotá, Colombia. \\ ${ }^{3}$ Centro de Geociencias, Universidad Nacional Autónoma de México, Campus Juriquilla, \\ Boulevard Juriquilla 3001, C.P. 76230, Querétaro, México. \\ ${ }^{4}$ Consultor independiente. Calle 113 No. 11A-55, Bogotá, Colombia. \\ ${ }^{5}$ Servicio Geológico Colombiano, Diagonal 53, No. 34 - 53, Bogotá D.C., Colombia. \\ * Grajales.jennyalejandra@gmail.com
}

\section{COMENTARIOS \\ SOBRE LA INTRODUCCIÓN}

En su comentario, Barbosa-Espitia y colaboradores dicen que se debió incluir en las referencias el trabajo de Barbosa-Espitia et al. (2019), como fuente original de los datos publicados en Grajales et al. (2020). Es necesario aclarar que los análisis en cuestión son propiedad del estado colombiano, hacen parte de los proyectos llevados a cabo por la Agencia Nacional de Hidrocarburos (ANH) y las firmas GRP S.A y el Consorcio Pacífico Colombiano, oficializado en el año 2014, y posteriormente esa información pasó a ser propiedad del Servicio Geológico Colombiano (SGC). En el año 2015 y mediante carta de la gerencia de Gestión del Conocimiento y de la Vicepresidencia Técnica, la ANH autorizó a la geóloga Alejandra Grajales el uso y publicación de los datos en cuestión, en el entorno de su tesis doctoral, de la cual es parte el artículo de Grajales et al. (2020). En ese sentido, se usó la referencia de autoría y propiedad original, el informe de la ANH GRP (2014). En el texto de Barbosa-Espitia et al. (2019), omiten esta última cita y hacen referencia a GRP (2011), cita que no es correcta en su temporalidad de oficialización, además de que eliminan a la ANH como el dueño y responsable de los resultados de ese proyecto.

\section{COMENTARIOS SOBRE EL POSIBLE ESCENARIO TECTÓNICO}

Ante el comentario sobre el uso de referencias previas, relacionadas a la presentación de un modelo de doble subducción que según Barbosa-Espitia et al. (2019) es considerado más plausible, los autores precisamos las razones por las cuáles no fue incluida como una referencia para el modelo presentado:
El objetivo de nuestro trabajo no es discutir las hipótesis sobre la evolución de placas tectónicas en el Pacífico colombiano. Nosotros presentamos como base de nuestra discusión la referencia original acerca del proceso de subducción a lo largo de la margen pacifica de Barrero (1979), la cual no fue incluída en el texto de Barbosa-Espitia et al. (2019). Nuestra interpretación se basó en las Juntas Triples (TJ, por sus siglas en inglés) que fueron asumidas en Barbosa-Espitia et al. (2019): una improbable de tipo TTT para el periodo 68-45 Ma, y para el periodo 45-39 Ma, una TJ improbable TTF de acuerdo con la clasificación de McKenzie y Morgan (1969). Tampoco aparece información sobre velocidades relativas entre las placas que permitan ilustrar las componentes de desplazamiento total para sustentar su hipótesis; además, su figura no tiene referencias de escala espacial para mostrar el tamaño de los eventos que ellos proponen. En realidad representan solamente esquemas geométricos conceptuales.

\section{EMPLAZAMIENTO DE INTRUSIVOS}

Barbosa-Espitia et al. (2019) critican nuestro trabajo arguyendo que "the contemporaneous Paleogene arc-like rocks from the PanamaChoco Block (northern WC) and southern WC have different origin and cannot be related on the basis of new and preexistent geochemical and isotopic data." Esto que dicen es muy extraño, ya que la base de nuestra argumentación es que hay continuidad física y contemporaneidad de los plutones, cosa que ellos mismos están admitiendo; entonces ¿qué tiene que ver la petrogénesis basada en composición química e isotópica? Al parecer Barbosa-Espitia et al. (2019) confundieron las variaciones composicionales con la ubicación físca y temporal de los magmas en discusión. Asi mismo, en relación al apartado "is inconsistent with the extensive magmatism in the Panama-Choco Block represented by the

Grajales, J.A., Nieto-Samaniego, Á.F., Barrero Lozano, D., Osorio, J.A., Cuellar, M.A., 2021, Resuesta al comentario de Barbosa-Espitia et al. a "Emplazamiento del magmatismo Paleoceno-Eoceno bajo un régimen transtensional y su evolución a un equilibrio dinámico en el borde occidental de Colombia”: Revista Mexicana de Ciencias Geológicas, v. 38, núm. 2, p. 148-149. 
Paleogene Mandé Batholith, which is a large tholeiitic-calc-alkaline pluton emplaced under low pressure conditions", es de aclarar a los autores del Comment, que las fracturas tensionales se forman por la disminución de la presión confinante en el sistema, por ende, el emplazamiento fue dado en condiciones de baja presión, por lo anterior, no es clara la inconsistencia a la que hacen alusión en el Comment.

Barbosa-Espitia et al. (2019) califican de "simplistas" las ideas de Zapata-García y Rodríguez-García (2020) que nosotros seguimos. Eso es una declaración poco afortunada, pues el modelo que califican de simplista permite explicar observaciones como las nuestras, y al parecer quieren minimizar su importancia solo por ser un modelo menos complejo que el de Barbosa-Espitia et al. (2019).

Con respecto al cese del arco magmático a partir de $45 \mathrm{Ma}$, Barbosa-Espitia et al. (2019) no presentan una explicación cinemática válida, pues no ilustran las consecuencias sobre los remanentes de placas oceánicas previamente en subducción, y no explican o discuten la formación de una sutura y sus consecuencias derivadas. Así mismo, no presentan ninguna interpretación alternativa sobre las velocidades de rollback generadas de manera particular en cada escenario propuesto para este complejo sistema de subducción.

Con respecto a la interpretación y uso de resultados de análisis geoquímicos, queremos comentar que no constituyen la parte central de la discusión y sus argumentos son interpretaciones que seguramente serán discutidos en trabajos dedicados a estudiar la química de esos magmas.

\section{COMPARACIÓN ENTRE MODELOS PALEOGEOGRÁFICOS Y TECTÓNICOS PROPUESTOS PREVIAMENTE}

En este apartado Barbosa-Espitia y colaboradores argumentan que hay numerosos datos de distinta índole que llevaron a formular un modelo "robusto" propuesto en estudios recientes por Cardona et al. (2018) y Barbosa-Espitia et al. (2019). Sin embargo, en ningún momento descartan o siquiera contradicen la argumentación fundamental del trabajo de Grajales et al. (2020), que es la continuidad del cinturón de rocas magmáticas, lo cual se expone con amplitud en el apartado "Tiempo de permanencia y migración del arco magmático" en Grajales et al. (2020). El objetivo de nuestro trabajo no es descartar los modelos existentes, sino mostrar datos que son mejor explicados por el modelo alternativo que se propone y que difícilmente son explicados por el modelo expuesto en Barbosa-Espitia et al. (2019).

En su argumentación, Barbosa-Espitia y colaboradores indican que nosotros proponemos que la subducción oblicua de la placa Farallón generó magmatismo simultáneo en las Cordilleras central y occidental, y argumentan que eso contradice datos termocronológicos, estructurales y paleomagnéticos de Colombia y Panamá, los cuales sugieren que el bloque Panamá-Chocó fue acrecionado al noroeste de Colombia durante el Neógeno. Nuevamente, Barbosa-Espitia y colaboradores yerran en su juicio, pues lo que nosostros presentamos son datos que muestran la contemporaneidad del evento magmático a la que ellos mismos hacen referencia y proponemos que el emplazamiento fue generado en un borde continental, previo a la evolución de un sistema de subducción. En todo caso, es el modelo presentado por Barbosa-Espitia et al. (2019) el que difícilmente puede explicar el magmatismo contemporáneo. Justo fue esa observación la que nos lleva a retomar algunas ideas que no siguen los modelos más recientes.
El argumento de continuidad del conjunto plutónico presentado en el apartado "Tiempo de permanencia y migración del arco magmático" en Grajales et al. (2020) no es cuestionado siquiera por Barbosa-Espitia y colaboradores. Eso lleva a la pregunta que inspiró el modelo alternativo propuesto: ¿Cómo se explica la continuidad del sistema magmático del Paleoceno-Eoceno en un esquema de bloques acrecionados incluso después del Eoceno?

Como conclusión podemos decir que, aunque seguramente se verá modificado conforme aparezca información nueva, el modelo altrnativo propuesto por Grajales et al. (2020) explica mejor la distribución y las edades del magmatismo.

\section{REFERENCIAS}

ANH-GRP (Agencia Nacional de Hidrocarburos, Geología Regional y Prospección), 2014, Cartografía geológica a escala 1:100.000 de las planchas 340, 362, 385 y 409 localizadas en la Cuenca de Tumaco, así como el inherente levantamiento de columnas estratigráficas y muestreo litológico para los análisis petrográficos, bioestratigráficos, geoquímicos, petrofísicos y radiométricos: Bogotá, Colombia, Agencia Nacional de Hidrocarburos, Publicación Especial, 207.

Barbosa-Espitia, A.A., Kamenov, G.D., Foster, D.A., Restrepo-Moreno, S.A. y Pardo-Trujillo, A. 2019, Contemporaneous Paleogene arc-magmatism within continental and accreted oceanic arc complexes in the north western Andes and Panama: Lithos, 348-349.

Barrero, D., 1979, Geology of the Central Western Cordillera, west of Buga and Roldanillo, Colombia: Publicaciones Geológicas especiales del Ingeominas, 4, 1-75.

Cardona, A., León, S., Jaramillo, J.S., Montes, C., Valencia, V., Vanegas, J., Bustamante, Echeverri, S., 2018, The Paleogene arcs of the northern Andes of Colombia and Panama: Insights on plate kinematic implications from new and existing geochemical, geochronological and isotopic data: Tectonophysics, 749, 88-103.

Grajales, J.A., Nieto-Samaniego, Á.F., Barrero Lozano, D., Osorio, J.A., Cuellar, M.A., 2020, Emplazamiento del magmatismo Paleoceno-Eoceno bajo un régimen transtensional y su evolución a un equilibrio dinámico en el borde occidental de Colombia: Revista Mexicana de Ciencias Geológicas, 37(3), 250-268.

McKenzie, D.P. y Morgan, W.J, 1969, Ecolution of Triple Junctions, Nature, 224, 125-133.

Zapata-García, G., Rodríguez-García, G., 2020, New contributions to the knowledge about the Chocó-Panamá Arc in Colombia, including a new segment south of Colombia, en Gómez, J., Mateus-Zabala, D. (eds.), The Geology of Colombia, Volume 3: Paleogene-Neogene: Servicio Geológico Colombiano, Publicaciones Geológicas Especiales 37, 34 pp.

Manuscrito recibido: marzo 22, 2021

Manuscrito aceptado: mayo 20, 2021 